\title{
How low can you grow?
}

Removal of the greenhouse gas methane by methanotrophic bacteria is an important climatic process. All methanotrophs characterized so far are meso- or thermophilic members of the phylum Proteobacteria. Now, two papers published in Nature report the cultivation, characterization and draft genome analysis of two new methanotrophs from the phylum Verrucomicrobia that are the most acidophilic methanotrophs ever studied.

Some environments that are rich in methane are also extremely acidic, so two groups of researchers set out to investigate whether novel methanotrophs thrive in these conditions. PCR primer sets for the conserved pmoA gene, which encodes a subunit of the particulate form of methane monooxygenase, have been used routinely to survey numerous environments for methanotrophs. Pol et al. extracted DNA from a hot, acidic, methaneproducing fumarole in the Solfatara, Italy, and used primers for $p m o A$ to construct an environmental clone library. They found two classes of pmoA sequences in the library - gammaproteobacterial-like sequences and a divergent set of pmoA homologues. Intrigued by these unusual $p m o A$ sequences, they isolated a strain into pure culture that had a divergent $p m o A$ sequence that was similar to those present in the clone library and a $16 \mathrm{~S}$ rRNA sequence that was typical of Verrucomicrobia, and named it SolV (provisional name Acidimethylosilex fumarolicum).

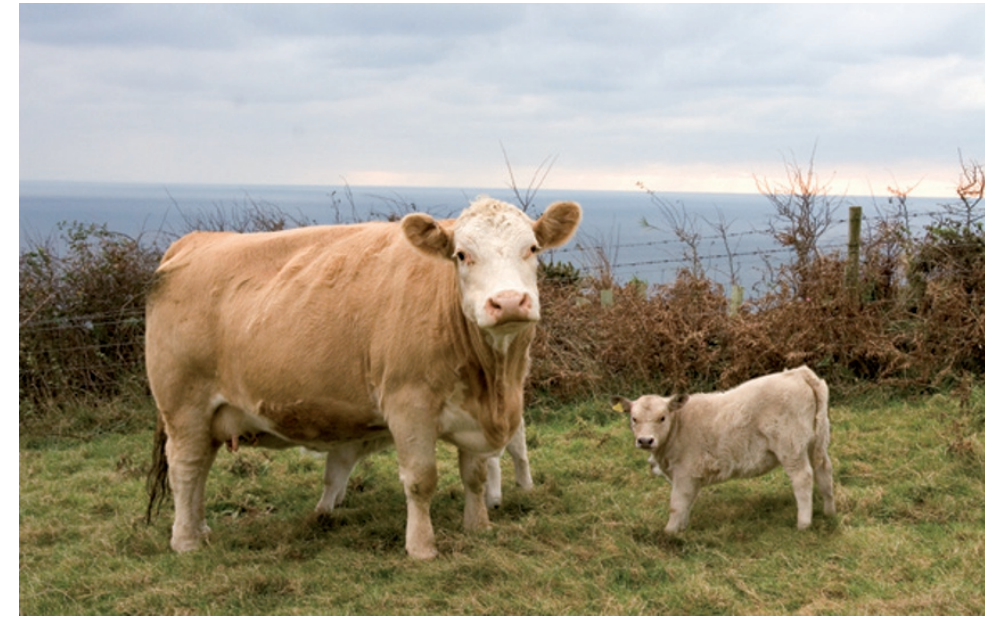

In a parallel study, Dunfield et al. analysed 16S rRNA genes of bacteria in a sample from Hell's Gate, an acidic geothermal site in New Zealand that is rich in methane, and identified novel Verrucomicrobia sequence signatures that were unrelated to any cultured bacteria. A strain named Verrucomicrobia isolate V4 (provisional name Methylokorus infernorum) was isolated into pure culture from this sample by supplying methane as the sole carbon and energy source. Both SolV and V4 have one striking phenotype: V4 can grow at $\mathrm{pH} 1.0$ and SolV can grow at $\mathrm{pH} 0.8$. This is the first time that extremely acidophilic methanotrophs have been described.

Draft genomes of both new species were assembled and scrutinized for insights into their physiologies; both are remarkable in that they have three pmoCAB operons that are completely different. Most proteobacterial methanotrophs typically encode up to three similar pmoCAB operons. Phylogenetic analyses of the three pmoA alleles encoded by V4 and those encoded by SolV indicate that these acidophilic lineages diverged from Proteobacteria a long time ago, and exclude the possiblity that Verrucomicrobia species have acquired the $p m o A$ gene by a recent horizontal-gene-transfer event. Inspection of the genomes indicated that both species might also use novel methylotrophic pathways.

These studies have provided an important step forward in the understanding of the physiology and diversity of methane consumption by bacteria in natural environments.

Susan Jones

ORIGINAL RESEARCH PAPERS Pol, A. et al. Methanotrophy below $\mathrm{pH} 1$ by a new

Verrucomicrobia species. Nature 14 Nov 2007 (doi 10.1038/nature06222)|Dunfield, P. F. et al. Methane oxidation by an extremely acidophilic bacterium of the phylum Verrucomicrobia. Nature 14 Nov 2007 (doi 10.1038/nature06411) 